\title{
On spectral efficiency maximization in a partial joint processing system using a multi- start particle swarm optimization algorithm
}

\begin{abstract}
Downlink joint processing (JP) between base stations eliminates the inter-cell interference in a cellular system with a frequency reuse factor of one and improves the spectral efficiency of cell-edge users. JP has a huge impact on both feedback and backhaul load, and thus partial JP was presented to tackle with signaling demand. However, achieving equivalent backhaul reduction based on limited feedback channel state information is challenging when linear techniques, such as zero-forcing beamforming (BF) are used, which led to the use of stochastic algorithms instead. Therefore stochastic multi-start particle swarm optimization algorithm (MSPSOA) is proposed in this paper to achieve backhaul reduction and address the issue of lack of diversity, which is related to the basic particle swarm optimization algorithm (BPSOA). The lack of diversity has been solved in this work by replacing the inactive particles adaptively based on a predefined constant which represents the difference between local best and global best optimization criterion. The performance of the proposed MSPSOA and BPSOA BF is evaluated with respect to full and partial JP using different metrics such as sum-rate, actual interference and convergence using a multipath realistic environment WINNER II channel model. The proposed MSPSOA outperforms BPSOA in terms of average sum-rate by $15.3 \%$, while the actual interference decreased by $14.6 \%$ in some conducted scenarios.
\end{abstract}

Keyword: Beamforming; Interference mitigation; Joint processing; Stochastic optimization; Swarm intelligence 